\title{
BMJ Open Protocol for a randomised trial of an interprofessional team-delivered intervention to support surrogate decision-makers in ICUs
}

\author{
Taylor Lincoln (D) , ${ }^{1}$ Anne-Marie Shields, ${ }^{2}$ Praewpannarai Buddadhumaruk, ${ }^{2}$ \\ Chung-Chou H Chang, ${ }^{3,4}$ Francis Pike, ${ }^{5}$ Hsiangyu Chen, ${ }^{6}$ Elke Brown, ${ }^{2}$ \\ Veronica Kozar, ${ }^{2}$ Caroline Pidro, ${ }^{4}$ Jeremy M Kahn, ${ }^{4}$ Joseph M Darby, ${ }^{4,7}$ \\ Susan Martin, ${ }^{8}$ Derek C Angus, ${ }^{4,7}$ Robert M Arnold, ${ }^{1,9}$ Douglas B White ${ }^{2,7}$
}

To cite: Lincoln T, Shields A$\mathrm{M}$, Buddadhumaruk $\mathrm{P}$, et al. Protocol for a randomised trial of an interprofessional team-delivered intervention to support surrogate decisionmakers in ICUs. BMJ Open 2020;10:e033521. doi:10.1136/ bmjopen-2019-033521

- Prepublication history for this paper is available online. To view these files, please visit the journal online (http://dx.doi. org/10.1136/bmjopen-2019033521).

Received 12 August 2019 Revised 06 January 2020 Accepted 03 February 2020

Check for updates

(C) Author(s) (or their employer(s)) 2020. Re-use permitted under CC BY-NC. No commercial re-use. See rights and permissions. Published by BMJ.

For numbered affiliations see end of article.

Correspondence to Dr Douglas B White; douglas.white@pitt.edu

\section{ABSTRACT}

Introduction Although shortcomings in clinician-family communication and decision making for incapacitated, critically ill patients are common, there are few rigorously tested interventions to improve outcomes. In this manuscript, we present our methodology for the Pairing Re-engineered Intensive Care Unit Teams with NurseDriven Emotional support and Relationship Building (PARTNER 2) trial, and discuss design challenges and their resolution.

Methods and analysis This is a pragmatic, steppedwedge, cluster randomised controlled trial comparing the PARTNER 2 intervention to usual care among 690 incapacitated, critically ill patients and their surrogates in five ICUs in Pennsylvania. Eligible subjects will include critically ill patients at high risk of death and/or severe long-term functional impairment, their main surrogate decision-maker and their clinicians. The PARTNER intervention is delivered by the interprofessional ICU team and overseen by 4-6 nurses from each ICU. It involves: (1) advanced communication skills training for nurses to deliver support to surrogates throughout the ICU stay; (2) deploying a structured family support pathway; (3) enacting strategies to foster collaboration between ICU and palliative care services and (4) providing intensive implementation support to each ICU to incorporate the family support pathway into clinicians' workflow. The primary outcome is surrogates' ratings of the quality of communication during the ICU stay as assessed by telephone at 6-month followup. Prespecified secondary outcomes include surrogates' scores on the Hospital Anxiety and Depression Scale, the Impact of Event Scale, the modified Patient Perception of Patient Centredness scale, the Decision Regret Scale, nurses' scores on the Maslach Burnout Inventory, and length of stay during and costs of the index hospitalisation. We also discuss key methodological challenges, including determining the optimal level of randomisation, using existing staff to deploy the intervention and maximising long-term follow-up of participants.

Ethics and dissemination We obtained ethics approval through the University of Pittsburgh, Human Research Protection Office. The findings will be published in peerreviewed journals.
Strengths and limitations of this study

- Use of existing clinical team in the study intensive care units (ICUs) to deploy the intervention.

- Multifaceted approach to maximise retention of participants for long-term outcome assessment.

- Need for in-person training of clinicians will necessitate a creative solution to allow broad dissemination.

- Absence of a 'gold standard' primary endpoint for interventions related to communication and decision making in ICUs.

Trial registration number NCT02445937

\section{INTRODUCTION}

Approximately one in five American deaths occur in or shortly after discharge from an intensive care unit (ICU). ${ }^{1}$ Many of these deaths occur following a decision to withhold or withdraw life-prolonging therapies. ${ }^{2-13}$ Because critically ill patients often lack decision-making capacity, surrogate decisionmakers are asked to assist in making these difficult decisions. A large body of research has documented problems with the process and outcomes of surrogate decision making in ICUs, including frequent failure to discuss patients' values, provide emotional support, hold timely family meetings or explain treatment options such as comfort-focused care. $^{14-18}$ Surrogates in ICUs perceive their role as challenging ${ }^{19-21}$ and experience high levels of depression, anxiety and posttraumatic stress disorder (PTSD). ${ }^{22} 23$ There is also evidence that critically ill patients often receive more intensive life-extending treatment than they would choose for themselves, which is problematic because of the impact 
on both individual patients and the costs of medical care near the end of life. ${ }^{2425}$

Although the scope of problems with surrogate decision making for critically ill patients is well documented, there are few evidence-based strategies to improve patient, family and health system outcomes in patients with advanced critical illness.

We, therefore, developed a multicomponent intervention delivered by the interprofessional ICU team to provide structured support of families throughout the ICU stay. ${ }^{26-28}$ The intervention is designed to follow national recommendations to use interdisciplinary teams to support patients and families ${ }^{29}{ }^{30}$ and leverage nurses' professional orientation toward providing patient and family-centred care. ${ }^{26-2831}$ The present trial differs from a previous trial of a similar intervention in several important ways. First, compared with the prior intervention tested, ${ }^{32}$ in the present trial, the scope of the PARNTER intervention was expanded to include a process to foster greater involvement of specialist palliative care (PC) clinicians into patients care and audit and feedback to be provided to each ICU. Second, the prior trial was completed with a small internal grant, which did not allow detailed collection of a variety of process outcomes, such as communication practices in both study arms and timing of decisions to limit the use of life-prolonging treatments or transition to comfort-focused goals of care. The present trial contains detailed data collection on these points. Third, the prior trial did not assess the impact of the intervention on bedside nurses which is included in the present trial's secondary outcomes. Herein, we present the trial methodology, describe the study intervention and discuss our approach to three key methodological challenges-determining level of randomisation, integrating the intervention with existing clinical staff and retaining participants in long-term follow-up.

\section{METHODS}

\section{Overview of trial methodology}

This is a pragmatic, stepped-wedge, cluster randomised controlled trial (RCT) evaluating the Pairing Re-engineered ICU Teams with Nurse-Driven Emotional Support and Relationship-Building (PARTNER) intervention compared with usual care control. We will assess the intervention's effect on three domains of outcomes: measures of the patient-centredness of care and quality of clinicianfamily communication, surrogates' symptoms of longterm psychological distress and healthcare utilisation.

\section{Trial centres and participants}

The trial will be conducted in five ICUs at four hospitals in Pennsylvania within the UPMC Health System: three medical ICUs within three community hospitals in which intensivist physicians serve as the attending physician of record for all patients, and a cardiac ICU and cardiothoracic surgical ICU within one academic hospital, in which intensivist physicians provide care for all patients in collaboration with a primary attending physician.

The trial will include all patients in the study ICUs who meet eligibility criteria during the enrolment period. Detailed inclusion and exclusion criteria are summarised in table 1 for patients, surrogate decision-makers and nurses. Inclusion criteria for patients include an age of 21 years or greater, lack of decision-making capacity and at least one of the following: receipt of mechanical

\begin{tabular}{|c|c|}
\hline \multicolumn{2}{|l|}{ Patient } \\
\hline Inclusion criteria & $\begin{array}{l}\text { Age } \geq 21 \text { years } \\
\text { Lack of decision-making capacity as determined by the clinical examination of the attending physician } \\
\text { At least one of the following: } \\
\text { 1. } \geq 96 \text { hours of mechanical ventilation. } \\
\text { 2. } \geq 40 \% \text { chance of hospital mortality as judged by the patient's attending physician. } \\
\text { 3. } \geq 40 \% \text { chance of severe long-term functional impairment as judged by the patient's attending } \\
\text { physician. }\end{array}$ \\
\hline Exclusion criteria & $\begin{array}{l}\text { Lack of surrogate decisions maker } \\
\text { Imminent organ transplantation }\end{array}$ \\
\hline \multicolumn{2}{|l|}{ Surrogate } \\
\hline Inclusion criteria & Clinical surrogate decision-maker, identified as the person making decisions for the patient. \\
\hline \multicolumn{2}{|l|}{ Clinician } \\
\hline Inclusion criteria & $\begin{array}{l}\text { PARTNER nurses (eg, nurse leaders, social workers). } \\
\text { Treating clinicians (eg, bedside nurses). }\end{array}$ \\
\hline Exclusion criteria & None \\
\hline
\end{tabular}

PARTNER, Pairing Re-Engineered ICU Teams with Nurse-Driven Emotional Support and Relationship-Building. 
ventilation for at least four consecutive days, judgement by the attending physician that the patient has at least a $40 \%$ chance of death during the hospitalisation or at least a $40 \%$ chance of severe long-term functional impairment. Daily a designated staff member in the ICU will screen the census and discuss each patient with the attending physician to determine eligibility for enrolment in the intervention pathway. Nurse leaders will identify one surrogate decision-maker per patient whom the family identifies as the patient's main surrogate and research staff will obtain their verbal consent for long-term follow-up over the phone. Nurses will be eligible if they were full time staff nurses in the ICU during the study period.

The study will be overseen by an independent data and safety monitoring committee (DSMC) consisting of three members with collective expertise in biostatistics, health services research, critical care medicine, behavioural interventions and bioethics. No interim analyses will be conducted. The DSMC will monitor patient accrual, retention and adverse events using a prespecified adverse event reporting protocol. The DSMC is empowered to stop the trial if evidence emerges of unexpectedly high rates of adverse events related to the intervention. Protocol amendments will be made in consultation with the trial's DSMC and the funding agency.

\section{Patient and public involvement}

Patients and the public were not directly involved in the design, recruitment or conduct of this study. However, research questions and outcomes measures were developed and informed by patients' and surrogates' priorities, experiences and preferences. ${ }^{14-18}$

\section{Description of the PARTNER Intervention}

The PARTNER intervention is conceptually grounded in the Cognitive Emotional Decision Making (CEDM) framework and Ottawa Decision Support Framework (ODSF) ${ }^{33} 34$ The CEDM framework views medical decisions as influenced by not only cognitive and informational considerations, but also the emotional distress that arises from witnessing a critically ill loved one and being required to make difficult, highly consequential decisions for them. The ODSF conceives that better patient/ family decision making can be achieved by (1) identifying decision support needs; (2) providing tailored decision support and (3) evaluating the decision-making process and outcomes. ${ }^{33} 35$

The PARTNER intervention will be deployed at the level of individual ICUs and delivered by the existing interprofessional ICU team. It will be overseen by four to six nurses from each ICU called the PARTNER nurses, nominated by their ICU director because they were judged to possess strong communication skills. The intervention entails guideline-recommended strategies for providing emotional support to surrogates and for ensuring frequent clinician-family communication. ${ }^{313637}$ The four main components, detailed below, are: (1) advanced communication skills training for 4-6 nurses from each
ICU to deliver support to surrogates throughout the ICU stay; (2) deploying a structured family support pathway delivered by the interprofessional ICU team (3) enacting strategies to increase the timely consultation of PC clinicians when appropriate and (4) providing comprehensive implementation support to ensure reliable delivery of the PARTNER intervention (table 2).

Advanced communication skills training for PARTNER nurses PARTNER nurses from each ICU will participate as a group in a 12-hour standardised, skills-focused training to develop the skills needed to support the surrogates of patients with advanced critical illness, summarised in table 2. The training programme for the PARTNER nurses adheres to the best practice recommendations from the National Institutes of Health (NIH) Behaviour Change Consortium. ${ }^{38}$ The teaching methods are grounded in principles of self-efficacy and adult learning theory ${ }^{39}$ and include: didactic explanation of the skill; demonstration by an expert clinician; small group practice with experienced medical actors portraying families and structured learner-centred feedback provided by an expert educator.

\section{Deploying a structured family support pathway delivered by the interprofessional ICU team}

After the nurses are trained in communication skills for the PARTNER intervention, each ICU will institute the family support pathway. The pathway involves the PARTNER nurses meeting with families daily, according to a standardised protocol, and arranging interdisciplinary clinician-family meetings within 48 hours after enrolment and every $5-7$ days thereafter. In addition, the PARTNER nurses meet with families before and after each family meeting to prepare them for the meeting and to debrief after the meeting. They also huddle with the clinical team before each family meeting. The main objectives of each encounter are summarised in table 2 and depicted in timeline format beginning from the day of enrolment in figure 1.

The PARTNER intervention uses strategies from behavioural economics and implementation science to overcome barriers to achieving frequent, structured family meetings. For example, the intervention resets the care default regarding timing of family meetings by switching from an 'opt-in' to an 'opt-out' approach to scheduling. Specifically, the PARTNER nurses will schedule family meetings per protocol unless the attending physician takes active steps to override the protocol, rather than requiring the clinical team to take active steps to schedule family meetings.

\section{Deploying strategies to increase collaboration between specialist PC and critical care services}

We will use three strategies: (1) identifying a specialist PC physician champion in each ICU to spearhead increased involvement of PC services; (2) facilitating a process in each ICU in which clinicians develop a set of suggested 'triggers' for PC consultation ${ }^{4041}$ and (3) conducting twice 
Table 2 Four components of the PARTNER intervention

1. Advanced communication skills training for 4-6 nurses from each ICU to deliver support to surrogates throughout the ICU stay

\begin{tabular}{|c|c|}
\hline Duration & 12 hours \\
\hline Teaching methods & $\begin{array}{l}\text { Didactic explanation of skills to be learnt } \\
\text { Demonstration of the skill by an expert clinician } \\
\text { Small group practice with simulated families } \\
\text { Learners receive feedback from and observe each other interact with simulated families } \\
\text { Structured-learner centred feedback provided by an expert communication skills } \\
\text { educator }\end{array}$ \\
\hline Ongoing training & Quarterly 'booster' training sessions in which key skills are reviewed and practised \\
\hline
\end{tabular}

2. Deploying a structured family support pathway delivered by interprofessional ICU team

First meeting with family Performs introduction

Provides emotional support using NURSE behaviours

Gets to know the family and the patient as individuals

Orients the family to the ICU

Before interdisciplinary meeting with family

Interdisciplinary meeting with family
Provides emotional support

Explains what to expect in the meeting

Elicits main concerns and completes question prompt list

Provides emotional support

Ensures that the family's main questions are answered

Brings the conversation back to the patient as an individual

Ensures that the treatment options are discussed

Ensures that there is a clear follow-up plan

After interdisciplinary meeting with the family

Attends to emotions raised during the meeting

Elicits questions

Corrects any misunderstandings of issues addressed during the meeting

Daily check-in

Check in daily to see how the family is doing

Updates the family on the plan for the day

Provides emotional support

Elicits questions and concerns

3. Enacting strategies to increase collaboration between ICU and PC services

Establishing a 'PC champion'

Provision of recommended 'triggers"* for PC consultation

Twice weekly, in-person meetings between PC and ICU services to review the ICU census

\section{Providing comprehensive implementation support to deploy the Intervention in each ICU}

Engagement of hospital and ICU Prior to implementation, study investigators sought explicit endorsement of the PARTNER leadership

Recruitment of PARTNER physician and nurse champions programme from hospital and ICU leadership at each site.

We will identify local nurse and critical care physician leaders at each site to act as a champion. These individuals commit to taking a leadership role for promoting the intervention and assisting with implementation challenges. 
Table 2 Continued

Orientation of all staff to the intervention

On-site implementation support
Study investigators will provide ICU physicians and bedside nurses with a structured orientation to the new care model and PARTNER nurses' role responsibilities via email communications and in-person education sessions.

During the first 2 weeks of deployment, an implementation specialist is on-site to provide daily assistance. Thereafter, the implementation specialist makes weekly visits to directly observe the clinicians deploying the intervention, provide feedback and assist in overcoming implementation challenges.

Audit-generated feedback on site performance of key process measures: no of patients enrolled, proportion who received IDFMs per protocol, frequency and timing of IDFMs compared with control phase, and frequency and timing of PC consults compared with control phase

${ }^{*}$ Proposed by expert working group, as summarised by Weissman and Meier ${ }^{40}$ and a suggested consensus-building strategy from the improving palliative care in the ICU (IPAL-ICU) working group. ${ }^{41}$

†Evidence-based strategies include the skills summarized in the NURSE mnemonic. ${ }^{68}$

ICU, intensive care unit; IDFM, interdisciplinary family meeting; PARNTER, Pairing Re-engineered ICU Teams with Nurse-Driven Emotional

Support and Relationship-Building; PC, palliative care.

weekly, in-person meetings between ICU and PC teams to assess whether any patients receiving the PARTNER intervention may benefit from a specialist PG consultation.

\section{Providing comprehensive implementation support}

The strategy for deployment is grounded in best practice recommendations to change clinician behaviour and enact system-level interventions. ${ }^{42}$ Our approach to implementation is informed by the theory of planned behaviour, which holds that success is determined by the strength of providers' motivation to engage and their perceived degree of control to implement the intervention, which is largely influenced by perceived self-efficacy and organisational factors. ${ }^{43} 44$

We will use five main techniques to encourage adherence throughout the study:

\section{Engagement of hospital and ICU leadership}

Prior to deployment at each site, study investigators will meet with hospital and ICU leadership to secure their endorsement of the PARTNER intervention. These leaders will send emails hospital-wide endorsing the intervention, as well as tailored emails to all ICU clinicians encouraging them to actively participate.

\section{Identification of PARTNER champions}

We will identify local nursing, critical care and PC champions in each ICU. These individuals will take on a leadership role for promoting the intervention and assisting with implementation challenges.

\section{Orientation of all staff to PARTNER intervention}

We will provide ICU physicians and bedside nurses with a structured orientation to the new care model and PARTNER nurses' role responsibilities.

\section{On-site implementation support}

During the first 2 weeks of deployment, an implementation specialist will be on-site to provide daily assistance. Thereafter, the implementation specialist makes weekly visits to directly observe the clinicians deploying the intervention, provide feedback and assist in overcoming implementation challenges.

\section{Quarterly audit and feedback}

The study team will provide each ICU with feedback on the extent to which the intervention is being deployed as planned with statistics summarising the number of patients enrolled, proportion who received family

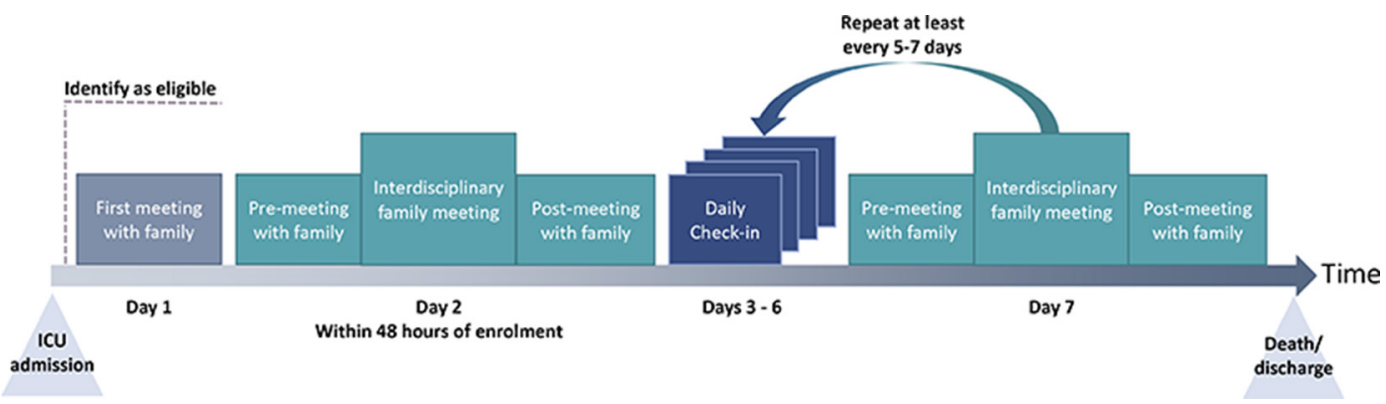

Figure 1 Family interaction with the PARTNER nurse in the family-support pathway. Timeline depicting each encounter between the PARTNER nurse and families in the family-support pathway beginning from the day of enrolment, noted as day 1. ICU, intensive care unit. 
Table 3 Randomisation results and the order of sites shifting to intervention phase with target timeline and accrual

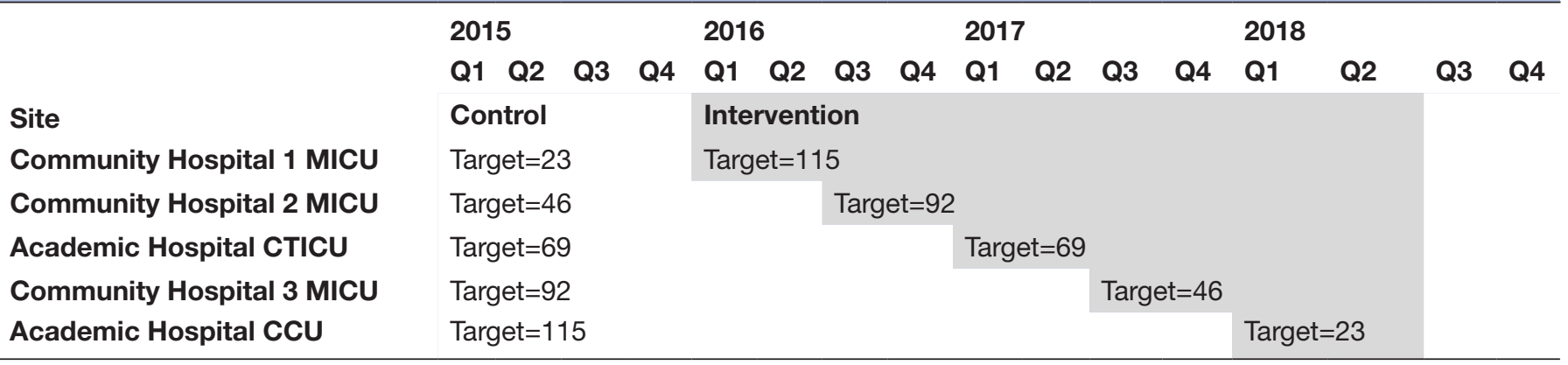

CCU, cardiac care unit; CTICU, cardiothoracic intensive care unit; MICU, medical intensive care unit.

meetings per protocol, frequency and timing of family meetings compared with control phase, and frequency and timing of PC consults compared with control phase.

\section{Description of usual care arm}

The control treatment consists of usual care. No study ICU has a protocolised approach to family communication or requires family meetings to be conducted at set times. At the time of the study, none of the ICUs will receive implementation support, audit or feedback related to family support and communication. PC consultation is available in all study ICUs.

\section{Randomisation}

The unit of randomisation is the individual ICU. The study statistician used a computer generated randomisation scheme to determine the order in which ICUs transition from the control phase to the intervention phase. Table 3 depicts randomisation results with targeted timeline and accrual rates. The plan is to complete enrolment in April 2019 and long-term follow-up in September 2019. All ICUs will receive the intervention by the end of the study period.

\section{Blinding}

The study staff performing chart abstraction and telephone follow-up of participants to ascertain study outcomes will be blinded to participants' treatment-group assignment. The nature of the intervention made it infeasible to mask physicians and surrogates to the patients' treatment-group assignment.

\section{Outcomes}

We developed an outcome assessment strategy to measure the effect of the intervention on three interrelated issues: the quality of communication (QOC) and patientcentredness and family centredness of care processes, surrogates' long-term psychological distress and healthcare utilisation and costs (table 4).

The primary outcome measure is surrogates' total score on the QOC scale, measured during telephone follow-up 6 months after patient's discharge from the index hospitalisation. The QOC Scale is a 13-item scale measuring QOC with good internal consistency, strong evidence of reliability and validity, ${ }^{45} 46$ and established responsiveness to change. ${ }^{47}$ The QOC scale is a patient and family-centred outcome because it measures aspects of care rated as highly important to patients and their families. Higher scores on the QOC scale have been associated with higher ratings of the patient-centredness of care, ${ }^{48}$ more goal concordant care and shorter duration of ICU care before death. ${ }^{32} 48$ We decided to focus long-term follow-up on only one time point in order to minimise the burden on family members, many of whom will be recently bereaved, and also to stay within budgetary constraints. We selected the 6-month time point because the QOC has established responsiveness to change at 6 months, ${ }^{32}$ and the secondary measures of psychological distress are of uncertain clinical significance prior to the 6-month time point.

The investigative team originally planned to use as the primary outcome surrogates' scores on the Hospital Anxiety and Depression Scale (HADS) at 6 months follow-up. However, the primary outcome was revised to the QOC scale and updated within ClinicalTrials.gov on 10 April 2018. The change in primary outcome measure was made in consultation with the trial's DSMC and the funding agency. The decision was based entirely on new evidence external to the trial. At no time did investigators have access to outcome data from the trial, which will not be made available to investigators until the trial is complete.

The rationale for this change was accumulating evidence that the type of intervention tested in this trial (ie, an intervention restricted to the ICU setting) is unlikely to improve surrogates' long-term symptoms of anxiety and depression. Two recently published RCTs of interventions focused on supporting family members acting as surrogates during their time in the ICU did not improve surrogates' psychological distress ${ }^{32}$ and may have worsened symptoms of PTSD. ${ }^{49}$ One of the interventions was very similar in design to the intervention being tested in the current trial. ${ }^{32}$

We will assess the following outcome measures through telephone interviews with surrogates at 6-month follow-up.

\section{Measures of communication and decision quality}

Patient-centredness and family-centredness of care: measured with the Patient Perception of Patient 
Table 4 Outcomes

\begin{tabular}{|c|c|c|c|c|}
\hline Domain & Outcomes & Instrument used & Data source & $\begin{array}{l}\text { Timing of } \\
\text { measurement }\end{array}$ \\
\hline \multicolumn{5}{|l|}{$\begin{array}{l}\text { Surrogate decision-maker } \\
\text { outcome }\end{array}$} \\
\hline \multirow{2}{*}{$\begin{array}{l}\text { Measures of } \\
\text { communication and } \\
\text { decision quality }\end{array}$} & $\begin{array}{l}\text { Quality of } \\
\text { communication (QOC) }\end{array}$ & QOC Scale* & Survey & $\begin{array}{l}\text { 6-month follow-up } \\
\text { from enrolment }\end{array}$ \\
\hline & Decisional regret & $\begin{array}{l}\text { Decisional Regret Scale } \\
\text { (DRS)‡ }\end{array}$ & Survey & $\begin{array}{l}\text { 6-month follow-up } \\
\text { from enrolment }\end{array}$ \\
\hline $\begin{array}{l}\text { Psychological symptoms } \\
\text { burden }\end{array}$ & Anxiety and depression & $\begin{array}{l}\text { Hospital Anxiety and } \\
\text { Depression Score (HADS)§ }\end{array}$ & Survey & $\begin{array}{l}6 \text {-month follow-up } \\
\text { from enrolment }\end{array}$ \\
\hline \multicolumn{5}{|l|}{ Healthcare costs } \\
\hline \multirow[t]{3}{*}{ Payer perspective } & $\begin{array}{l}\text { Index hospitalisation } \\
\text { cost }\end{array}$ & & $\begin{array}{l}\text { Hospital billing } \\
\text { records }\end{array}$ & Postdischarge \\
\hline & $\begin{array}{l}\text { Postdischarge } \\
\text { healthcare utilisation }\end{array}$ & & $\begin{array}{l}\text { Hospital billing } \\
\text { records, medical } \\
\text { records and } \\
\text { surrogate interview }\end{array}$ & $\begin{array}{l}\text { 6-month follow-up } \\
\text { from enrolment }\end{array}$ \\
\hline & $\begin{array}{l}\text { Hospital readmission } \\
\text { rates }\end{array}$ & & Surrogate & $\begin{array}{l}\text { 6-month follow-up } \\
\text { from enrolment }\end{array}$ \\
\hline Hospital perspective & $\begin{array}{l}\text { Index hospitalisation } \\
\text { costs }\end{array}$ & & $\begin{array}{l}\text { UPMC health } \\
\text { systems' } \\
\text { Computerised cost } \\
\text { accounting system }\end{array}$ & Postdischarge \\
\hline \multicolumn{5}{|l|}{ Patient-centred outcomes } \\
\hline & $\begin{array}{l}\text { Discharge disposition } \\
\text { (including in hospital } \\
\text { mortality) }\end{array}$ & & $\begin{array}{l}\text { Registration data, } \\
\text { chart abstraction }\end{array}$ & Postdischarge \\
\hline & $\begin{array}{l}\text { Functional status at } 6 \\
\text { months }\end{array}$ & Katz ADL ${ }^{* *}$ & Surrogate & $\begin{array}{l}6 \text {-month follow-up } \\
\text { from enrolment }\end{array}$ \\
\hline & $\begin{array}{l}\text { Living situation at } 6 \\
\text { months }\end{array}$ & & $\begin{array}{l}\text { 6-month follow-up } \\
\text { with surrogates }\end{array}$ & $\begin{array}{l}\text { 6-month follow-up } \\
\text { from enrolment }\end{array}$ \\
\hline & $\begin{array}{l}\text { All-cause 6-month } \\
\text { mortality }\end{array}$ & & $\begin{array}{l}\text { Hospital records, } \\
6 \text {-month follow-up } \\
\text { with surrogates and } \\
\text { the National Death } \\
\text { Index }\end{array}$ & $\begin{array}{l}\text { 6-month follow-up } \\
\text { from enrolment }\end{array}$ \\
\hline
\end{tabular}


Table 4 Continued

\begin{tabular}{|c|c|c|c|c|}
\hline Domain & Outcomes & Instrument used & Data source & $\begin{array}{l}\text { Timing of } \\
\text { measurement }\end{array}$ \\
\hline & Clinician burn-out & $\begin{array}{l}\text { Maslach Burn-out } \\
\text { Inventory†† }\end{array}$ & $\begin{array}{l}\text { Bedside nurses } \\
\text { caring for patients } \\
\text { enrolled in the study }\end{array}$ & $\begin{array}{l}\text { Baseline, } 6 \text { months } \\
\text { after randomisation }\end{array}$ \\
\hline \multicolumn{5}{|c|}{ Process measures } \\
\hline & $\begin{array}{l}\text { Frequency of } \\
\text { multidisciplinary } \\
\text { communication }\end{array}$ & & Chart abstraction & Postdischarge \\
\hline & $\begin{array}{l}\text { Palliative care and } \\
\text { ethics consultations }\end{array}$ & & Chart abstraction & Postdischarge \\
\hline & $\begin{array}{l}\text { Social work } \\
\text { involvement }\end{array}$ & & Chart abstraction & Postdischarge \\
\hline
\end{tabular}

${ }^{*} \mathrm{QOC}$ is a 13-item scale measuring QOC with good internal consistency (alpha=0.94), strong evidence of reliability and validity ${ }^{45} 46$ and established responsiveness to change.

TPPPC is a 12-item instrument that measures the patient-centredness of care and has demonstrated validity and reliability when used by surrogates. (Cronbach's $\alpha=0.71)^{50}$ A recent systematic review found the PPPC to be one of two best instruments to measure this construct. ${ }^{51}$ fDRS is a 5-item assessment of 'distress or remorse after healthcare decisions.' It has high internal consistency and convergent validity. ${ }^{69}$ §HADS is a 14-item assessment with subscales for anxiety and depression. Each domain has a score range of 0-21 with the following interpretation: $0-7$ normal, 8-10 borderline abnormal and 11-21, abnormal.

qIES is a 15-item tool measuring total stress (score range of $0-75$ ) with subscales for intrusiveness (score range $0-35$ ) and avoidance (score range 0-40). Total stress score is interpreted as follows: 0-8 subclinical range, 9-25 mild range, 26-43 moderate range, and 44+severe range. A score of $\geq 30$ indicates a high risk of post-traumatic stress disorder. The IES is a valid, reliable and responsive 15-item instrument measuring symptoms of avoidance and intrusive thoughts. ${ }^{59}$ It has been successfully used among ICU surrogates. ${ }^{22} 27$

${ }^{* *}$ Katz ADL.

††Maslach Burnout Inventory is a validated, widely used measure of clinician burnout. ${ }^{70-72}$

Centredness (PPPC) scale, modified for use by surrogates. The PPPC is a 12-item instrument that has established validity and reliability. ${ }^{50}$

Decisional regret: measured with the Decisional Regret Scale, a 5-item assessment of 'distress or remorse after healthcare decisions.' It has high internal consistency and convergent validity. ${ }^{52}$

\section{Surrogates' psychological distress}

Anxiety and depressive symptoms: The HADS is a 14-item, two-domain instrument used to study anxiety and depression with established reliability and validity among ICU surrogates. ${ }^{27} 53-58$

Symptoms of PTSD: The Impact of Events Scale is a 15-item tool measuring total stress with subscales for intrusiveness and avoidance ${ }^{59}$ It has been successfully used among ICU surrogates. ${ }^{22} 27$

\section{Patients' outcomes}

Discharge disposition: We will use University of Pittsburgh Medical Center (UPMC) administrative records to determine if patients were discharged to home, hospice, a skilled nursing facility, another acute care hospital and a long-term acute care facility.

Mortality: We will assess mortality during the index hospitalisation and through 6-month follow-up using hospital records, telephone interviews with surrogates at 6-month follow-up, and the Social Security Death Master File in cases if participants are lost to telephone follow-up.

Functional status at 6 months: We will assess patients' functional status at 6 months after discharge using the Katz Activities of Daily Living (ADL) instrument completed by surrogates during the 6-month follow-up call. ${ }^{60}$

Healthcare utilisation and costs

ICU and hospital length of stay: We will determine the intervention's impact on patients' ICU and hospital length of stay, measured from study enrolment using the UPMC electronic medical record.

Total hospitalisation costs: We will measure costs during the index hospitalisation using the UPMC computerised cost accounting system, which assigns specific costs to each service based on hospital expenses. UPMC developed this activity-based costing (ABC) system to align costs with patients based on actual utilisation of resources. Direct expenses, such as blood products, drugs and supplies, are allocated on a patient-incurred basis. Departmental labour and other expenses are allocated to patients using specific cost drivers, such minutes on a nursing unit or time in an OR. This costing method excludes expenses related to physician margin (Physician Services Division 
Sweep and Support), Enterprise Shared Services and other fully indirect expenses. These excluded categories total approximately $25 \%$ of total hospital expenses. Because this costing system excludes these fixed costs, the costs may alternatively be labelled 'total controllable hospitalisation costs'. Further details of the ABC system can be found at: https://www.healthcatalyst. com/success_stories/activitybased-costing-in-healthcareservice-lines-upmc. To calculate direct variable costs, we will remove the fixed costs of overhead that are not related to patient throughput, determined through individual departmental usage patterns and will aggregate each patient's total service specific costs. ${ }^{61}$

Healthcare utilisation through 6-month follow-up: We will measure healthcare utilisation that occurs between index hospitalisation discharge and 6-month follow-up using an established method based on in-depth interview with the patient's surrogate during the 6-month follow-up call. ${ }^{62}$ The interview contains questions to determine the number of postdischarge hospital admissions, nursing home admissions, emergency department visits, physician visits, hospice use and home health service utilisation.

Cost of Implementing PARTNER Intervention: To calculate costs to implement the intervention, we will determine the cost of all training, inclusive of salary/ fringe costs of nurses, actors and instructors. We will also include costs related to ongoing implementation support during the intervention phase of the study, including the implementation specialists' time, mileage and parking for travel between sites.

\section{Statistical methods}

All analyses will be performed on an intention-to-treat basis. The individual ICU is the unit of randomisation and individual surrogate/patient is the unit of analysis. To compare surrogates' characteristics across treatment groups, we will use t-tests or Wilcoxon rank-sum tests for continuous outcomes and $\mathrm{X}^{2}$ or Fisher's exact tests for categorical outcomes. To determine whether the intervention impacts QOC scores (as well as other continuous secondary outcomes), we will use generalised linear mixed model to account for temporal and clustering effects typically encountered in stepped-wedge clinical trial designs. ${ }^{63}{ }^{64}$ Clustering effects by ICU will be treated as random in the models. We will include time as fixed effects to account for secular trends over time and include time by treatment interaction to investigate whether treatment effect is time-varying. The model will also include random slopes of time to account for possible heterogeneous temporal effects across ICUs.

A known limitation of the stepped-wedge cluster RCT design is that there are often imbalances in patient characteristics across study sites that site-level randomisation does not address. Therefore, it is an accepted strategy in stepped-wedge trials to prespecify that certain covariates will be adjusted for in the statistical analysis plan. We will, therefore, adjust analyses for patient age, modified Simplified Acute Physiology Score (SAPS) III, Elixhauser index, mechanical ventilation usage and admission source. In addition, if there are baseline differences between other demographic characteristics across treatment arms, we will adjust for those associated with the outcome in univariate analysis with a $\mathrm{p}$ value less than or equal to 0.20 . Should missing data prove problematic we will use the methods of multiple imputations or inverse probability weighting. ${ }^{65}$ Final models will be assessed for stability using routine model diagnostics to identify potential outliers and/or influential observations.

Sample size determination. With a sample size of 690 surrogates, assuming 20\% lost to follow-up and alpha $=0.05$, we will have $80 \%$ power to detect a small effect size difference (Cohen's d: 0.30) between groups on the QOC scale. The MCID for the QOC scale has not been established, but differences between groups of this magnitude were observed in a recent trial of a family support intervention in ICUs and were associated with improved ratings of patient-centredness and familycentredness of care (measured with the modified PPPC scale), as well as a shortened ICU and hospital length of stay among dying patients. ${ }^{66}$ In addition, a recent trial of an intervention to improve communication about goals of care yielded significant improvements in the QOC with a Cohen's $d$ of 0.56 , which were in turn associated with improved rating of goal-concordant care among patients with stable goals through 3-month follow-up. ${ }^{67}$

The power calculation was done via NCSS PASS 15 using the pooled outcome $(\mathrm{SD}=24.5)$ and coefficient of variation (0.15) from the Family-Support Intervention in Intensive Care Units study. ${ }^{48}$ We assumed a two-sided alpha level of 0.05 and a conservative estimate of $20 \%$ for the rate of surrogate lost to follow-up.

The enrolment goal was initially 1000 and changed to 690 after a senior biostatistician joined the study team. We re-examined initial power calculations and concluded we were overpowered for our main outcome measure and any gains from obtaining a larger sample size would be only incremental. For practical reasons, because of recent decreases in ICU admissions to UPMC hospitals due to a split between UPMC and Highmark, one of the main health insurance companies, we determined that enrolment would likely be slower than we originally predicted. Given the nature of stepped-wedge trials (ie, control subjects enrolled first, then intervention subjects later) there was concern slow enrolment could led to a circumstance in which too few intervention patients are enrolled.

\section{Ethics and dissemination}

Research ethics approval

The institutional review board of the University of Pittsburgh and the quality improvement committee of the UPMC Health System approved the project. The leadership of each participating ICU approved the project. The intervention was judged to be a quality improvement initiative. Surrogates of eligible patients were informed of the QI project by ICU staff, however, consent was not 
required for enrolment in the intervention pathway. The long-term follow-up of surrogates and nurses was judged to be research. Nurse leaders within each ICU will identify a surrogate decision-maker and introduce the research study. If family members give permission for the research staff to contact them, forms will be faxed or emailed via secure server behind the institutional firewall to the research staff who then attempt to call within 48 hours. If the family member is willing to participate, we continue with the verbal consent process over the phone. Nurses will provide written informed consent for their participation.

\section{Dissemination}

We will make results available to surrogate decisionmakers and caregivers, the funders, critical care societies and other researchers. We will use traditional methods, including presentation at national meetings, submission to peer-reviewed journals and use of social media to disseminate findings.

\section{DISCUSSION}

While planning the trial, we identified three key design and implementation challenges: (1) determining the optimal type of randomised trial design, (2) using existing clinical staff to deploy the intervention and (3) maximising long-term follow-up.

We chose to use a stepped-wedge cluster randomised design rather than an individual-level RCT or a cluster RCT for two main reasons. First, we judged that randomising individual patients within ICUs would create a high risk of contamination of the control arm because the intervention is deployed at the ICU-level. Second, a cluster RCT would involve randomly assigning half of the ICUs to receive the intervention and half to receive the control for the duration of the study. Individual ICUs were unwilling to be randomised to a control condition for the duration of the study because of mounting societal pressure to improve end-of-life care for patients with advanced critical illness. We ultimately selected a stepped-wedge cluster randomised design because it allows randomization at the ICU-level and allows all ICUs to receive the intervention during the study period.

We elected to use existing clinical staff to deploy the intervention for two main reasons. First, doing so increases the scalability of the intervention compared with either using research personnel to deliver the intervention or adding additional clinical personnel to the ICU care team to deliver the intervention. Second, we hypothesise that achieving durable improvements in family support will require changing ICUs' overall culture and processes of care, which may be more likely to occur when the intervention targets the entire interprofessional team rather than external interventionists.

Deploying the PARTNER intervention through the existing interprofessional team also presents several challenges. First, ICU clinicians are busy and the PARTNER intervention will likely result in an increase in the amount of time devoted to clinician-family communication. We address this by providing on-site support to develop efficient care processes and training multiple PARTNER nurses per unit. Second, few front-line clinicians have experience deploying complex, protocolised behavioural interventions, which may pose threats to intervention fidelity. We addressed the potential issue by developing a rigorous training programme that focuses on the need for high adherence to protocol. In addition, we designed an extensive monitoring programming that involves weekly site visits with direct observation and coaching by implementation specialists, quarterly 'booster' training sessions in which key communication skills are reviewed, and quarterly audit and feedback sessions where unit adherence is summarised.

The third challenge is achieving adequately high rates of long-term follow-up. Long-term follow-up can be challenging in this population because most of the participants will either be recently bereaved or will be caregivers for survivors of critical illness. We developed three strategies to maximise retention during the follow-up period. First, we collect extensive contact information at initial consenting including phone numbers, mailing addresses and email addresses of both the patient's surrogate and an alternate contact who will know how to contact the participant if the surrogate's contact information is no longer valid. Second, we seek to maintain contact with the surrogate after hospital discharge beginning with a thank you note following the consenting process. We also send participants a letter 4-month posthospitalisation with information on scheduling the 6-month follow-up call at their convenience. Third, we developed a protocol for subjects who were hard-to-reach outlining appropriate use of retention strategies, such as voicemail, a hard-to-reach letter, mail return service requesting, use of the alternate contact, online searches for new information and a version of the follow-up interview to complete via mail. We ensure these protocols are implemented through use of software with detailed record of all follow-up activities.

\section{Author affiliations}

${ }^{1}$ Department of General Internal Medicine, Section of Palliative Care and Medical Ethics, University of Pittsburgh School of Medicine, Pittsburgh, Pennsylvania, USA ${ }^{2}$ Department of Critical Care Medicine, The CRISMA Center, Program on Ethics and Decision Making, University of Pittsburgh School of Medicine, Pittsburgh, Pennsylvania, USA

${ }^{3}$ Department of Biostatistics, University of Pittsburgh Graduate School of Public Health, Pittsburgh, Pennsylvania, USA

${ }^{4}$ Department of Critical Care Medicine, The CRISMA Center, University of Pittsburgh School of Medicine, Pittsburgh, Pennsylvania, USA

${ }^{5}$ Department of Neuroscience, Ely Lilly and Company, Indianapolis, Indiana, USA ${ }^{6}$ Center for Clinical Epidemiology and Biostatistics, University of Pennsylvania Perelman School of Medicine, Philadelphia, Pennsylvania, USA

${ }^{7}$ ICU Service Center, UPMC Health System, Pittsburgh, Pennsylvania, USA

${ }^{8}$ Donald Wolff Center for Quality Improvement and Innovation, UPMC Health System, Pittsburgh, Pennsylvania, USA

${ }^{9}$ Palliative Support Institute, UPMC Health System, Pittsburgh, Pennsylvania, USA

Contributors TL: Drafting the work and revisiting it critically for important intellectual content. Final approval of the version to be published. A-MS: Substantial 
contributions to the acquisition of data for the work. Revising the work critically for important intellectual content. Final approval of the version to be published. PB: Substantial contributions to the trial design and analytic plan. C-CHC: Substantial contributions to the trial design and analytic plan. Revising the work critically for important intellectual content. Final approval of the version to be published. FP: Substantial contributions to the conception or design of the work. Revising the work critically for important intellectual content. Final approval of the version to be published. HC: Substantial contributions to the conception or design of the work. Revising the work critically for important intellectual content. Final approval of the version to be published. EB: Substantial contributions to the conception or design of the work. Revising the work critically for important intellectual content. Final approval of the version to be published. VK: Substantial contributions to acquisition of data for the work. Revising the work critically for important intellectual content. Final approval of the version to be published. CP: Substantial contributions to the conception or design of the work. Revising the work critically for important intellectual content. Final approval of the version to be published. JMK: Substantial contributions to the conception or design of the work. Revising the work critically for important intellectual content. Final approval of the version to be published. JMD: Substantial contributions to the conception or design of the work. Revising the work critically for important intellectual content. Final approval of the version to be published. SM: Substantial contributions to the conception or design of the work. Revising the work critically for important intellectual content. Final approval of the version to be published. DCA: Substantial contributions to the conception or design of the work. Revising the work critically for important intellectual content. Final approval of the version to be published. RMA: Substantial contributions to the conception or design of the work. Revising the work critically for important intellectual content. Final approval of the version to be published. DBW: Substantial contributions to the conception or design of the work. Drafting the work and revisiting it critically for important intellectual content. Final approval of the version to be published. Agreement to be accountable for all aspects of the work in ensuring that questions related to the accuracy or integrity of any part of the work are appropriately investigated and resolved.

Funding This work was supported by DBW, NIH/ National Institute of Nursing Research (NINR) grant number R01NR014663.

Competing interests None declared.

Patient consent for publication Not required.

Provenance and peer review Not commissioned; externally peer reviewed.

Open access This is an open access article distributed in accordance with the Creative Commons Attribution Non Commercial (CC BY-NC 4.0) license, which permits others to distribute, remix, adapt, build upon this work non-commercially, and license their derivative works on different terms, provided the original work is properly cited, appropriate credit is given, any changes made indicated, and the use is non-commercial. See: http://creativecommons.org/licenses/by-nc/4.0/.

ORCID iD

Taylor Lincoln http://orcid.org/0000-0002-9327-0290

\section{REFERENCES}

1 Angus DC, Barnato AE, Linde-Zwirble WT, et al. Use of intensive care at the end of life in the United States: an epidemiologic study. Crit Care Med 2004;32:638-43.

2 Prendergast TJ, Claessens MT, Luce JM. A national survey of end-of-life care for critically ill patients. Am J Respir Crit Care Med 1998;158:1163-7.

3 Bedell SE, Pelle D, Maher PL, et al. Do-Not-Resuscitate orders for critically ill patients in the hospital. how are they used and what is their impact? JAMA 1986;256:233-7.

4 Eidelman LA, Jakobson DJ, Pizov R, et al. Foregoing life-sustaining treatment in an Israeli ICU. Intensive Care Med 1998;24:162-6.

5 Faber-Langendoen $\mathrm{K}$. The clinical management of dying patients receiving mechanical ventilation. A survey of physician practice. Chest 1994;106:880-8.

6 Keenan SP, Busche KD, Chen LM, et al. A retrospective review of a large cohort of patients undergoing the process of withholding or withdrawal of life support. Crit Care Med 1997;25:1324-31.

7 Koch KA, Rodeffer HD, Wears RL. Changing patterns of terminal care management in an intensive care unit. Crit Care Med 1994;22:233-43.

8 Smedira NG, Evans BH, Grais LS, et al. Withholding and withdrawal of life support from the critically ill. N Engl J Med 1990;322:309-15.
9 Vernon DD, Dean JM, Timmons OD, et al. Modes of death in the pediatric intensive care unit: withdrawal and limitation of supportive care. Crit Care Med 1993;21:1798-802

10 Vincent JL, Parquier JN, Preiser JC, et al. Terminal events in the intensive care unit: review of 258 fatal cases in one year. Crit Care Med 1989;17:530-3.

11 Youngner SJ, Lewandowski W, McClish DK, et al. 'Do not resuscitate' orders. incidence and implications in a medical-intensive care unit. JAMA 1985;253:54-7.

12 Sprung CL, Cohen SL, Sjokvist P, et al. End-Of-Life practices in European intensive care units: the Ethicus study. JAMA 2003;290:790-7.

13 Prendergast TJ, Luce JM. Increasing incidence of withholding and withdrawal of life support from the critically ill. Am J Respir Crit Care Med 1997;155:15-20.

14 Scheunemann LP, Cunningham TV, Arnold RM, et al. How clinicians discuss critically ill patients' preferences and values with surrogates: an empirical analysis. Crit Care Med 2015;43:757-64.

15 Selph RB, Shiang J, Engelberg R, et al. Empathy and life support decisions in intensive care units. J Gen Intern Med 2008;23:1311-7.

16 White DB, Engelberg RA, Wenrich MD, et al. Prognostication during physician-family discussions about limiting life support in intensive care units. Crit Care Med 2007;35:442-8.

17 White DB, Braddock CH, Bereknyei S, et al. Toward shared decision making at the end of life in intensive care units: opportunities for improvement. Arch Intern Med 2007;167:461-7.

18 White DB, Engelberg RA, Wenrich MD, et al. The language of prognostication in intensive care units. Med Decis Making 2010;30:76-83.

19 Kirchhoff KT, Walker L, Hutton A, et al. The vortex: families' experiences with death in the intensive care unit. Am J Crit Care 2002;11:200-9.

20 Tilden VP, Tolle SW, Garland MJ, et al. Decisions about life-sustaining treatment. impact of physicians' behaviors on the family. Arch Intern Med 1995;155:633-8.

21 Tilden VP, Tolle SW, Nelson CA, et al. Family decision-making to withdraw life-sustaining treatments from hospitalized patients. Nurs Res 2001;50:105-15

22 Azoulay E, Pochard F, Kentish-Barnes N, et al. Risk of post-traumatic stress symptoms in family members of intensive care unit patients. Am J Respir Crit Care Med 2005;171:987-94.

23 Wendler D, Rid A. Systematic review: the effect on surrogates of making treatment decisions for others. Ann Intern Med 2011;154:336-46.

24 A controlled trial to improve care for seriously ill hospitalized patients. the study to understand prognoses and preferences for outcomes and risks of treatments (support). The support principal Investigators. JAMA 1995;274:1591-8.

25 Riley GF, Lubitz JD. Long-Term trends in Medicare payments in the last year of life. Health Serv Res 2010;45:565-76.

26 Schneiderman LJ, Gilmer T, Teetzel HD, et al. Effect of ethics consultations on nonbeneficial life-sustaining treatments in the intensive care setting: a randomized controlled trial. JAMA 2003;290:1166-72.

27 Lautrette A, Darmon M, Megarbane B, et al. A communication strategy and brochure for relatives of patients dying in the ICU. $N$ Engl J Med 2007;356:469-78.

28 Curtis JR, Nielsen EL, Treece PD, et al. Effect of a qualityimprovement intervention on end-of-life care in the intensive care unit: a randomized trial. Am J Respir Crit Care Med 2011;183:348-55.

29 Institute of Medicine (US) Committee on Quality of Health Care in America. Crossing the quality chasm: a new health system for the 21st century. Washington (DC), 2001.

30 Field MJ, Cassel CK, eds. Approaching Death: Improving Care at the End of Life. Washington (DC), 1997.

31 Davidson JE, Aslakson RA, Long AC, et al. Guidelines for FamilyCentered care in the neonatal, pediatric, and adult ICU. Crit Care Med 2017;45:103-28.

32 White DB, Angus DC, Shields A-M, et al. A randomized trial of a Family-Support intervention in intensive care units. N Engl J Med 2018;378:2365-75.

33 Légaré F, O'Connor AC, Graham I, et al. Supporting patients facing difficult health care decisions: use of the Ottawa decision support framework. Can Fam Physician 2006;52:476-7.

34 Power TE, Swartzman LC, Robinson JW, et al. Cognitive-emotiona decision making (CEDM): a framework of patient medical decision making. Patient Educ Couns 2011;83:163-9.

35 Murray MA, Miller T, Fiset V, et al. Decision support: helping patients and families to find a balance at the end of life. Int $J$ Palliat Nurs 2004;10:270-7. 
36 Kon AA, Davidson JE, Morrison W, et al. Shared decision making in ICUs: an American College of critical care medicine and American thoracic Society policy statement. Crit Care Med 2016;44:188-201.

37 Truog RD, Campbell ML, Curtis JR, et al. Recommendations for end-of-life care in the intensive care unit: a consensus statement by the American College [corrected] of Critical Care Medicine. Crit Care Med 2008;36:953-63.

38 Bellg AJ, Borrelli B, Resnick B, et al. Enhancing treatment fidelity in health behavior change studies: best practices and recommendations from the $\mathrm{NIH}$ behavior change Consortium. Health Psychol 2004;23:443-51.

39 Rimer BK, Glanz K. Theory at a glance: a guide for health promotion practice. Bethesda, MD: National Institute of Health, National Cancer Institute, 2005.

40 Weissman DE, Meier DE. Identifying patients in need of a palliative care assessment in the hospital setting: a consensus report from the center to advance palliative care. J Palliat Med 2011;14:17-23.

41 Nelson JE, Curtis JR, Mulkerin C, et al. Choosing and using screening criteria for palliative care consultation in the ICU: a report from the improving palliative care in the ICU (IPAL-ICU) Advisory board. Crit Care Med 2013;41:2318-27.

42 Grol R, Grimshaw J. From best evidence to best practice: effective implementation of change in patients' care. Lancet 2003;362:1225-30.

43 Ajzen I. The theory of planned behavior. Organ Behav Hum Decis Process 1991;50:179-211.

44 Reviews ATShojania KG, McDonald KM, Wachter RM, eds. Closing the quality gap: a critical analysis of quality improvement strategies (vol. 1: series overview and methodology). Rockville (MD: Agency for Healthcare Research and Quality (US), 2004.

45 Curtis JR, Patrick DL, Caldwell E, et al. The quality of patientdoctor communication about end-of-life care: a study of patients with advanced AIDS and their primary care clinicians. AIDS 1999:13:1123-31.

46 Engelberg R, Downey L, Curtis JR. Psychometric characteristics of a quality of communication questionnaire assessing communication about end-of-life care. J Palliat Med 2006;9:1086-98.

$47 \mathrm{Au} \mathrm{DH}$, Udris EM, Engelberg RA, et al. A randomized trial to improve communication about end-of-life care among patients with COPD. Chest 2012;141:726-35.

48 Curtis JR, Downey L, Back AL, et al. Effect of a patient and clinician Communication-Priming intervention on patient-reported Goals-ofCare discussions between patients with serious illness and clinicians: a randomized clinical trial. JAMA Intern Med 2018;178:930-40.

49 Carson SS, Cox CE, Wallenstein S, et al. Effect of palliative CareLed meetings for families of patients with chronic critical illness: a randomized clinical trial. JAMA 2016;316:51-62.

50 Stewart M, Brown JB, Donner A, et al. The impact of patientcentered care on outcomes. J Fam Pract 2000;49:796-804

51 Hudon C, Fortin M, Haggerty JL, et al. Measuring patients perceptions of patient-centered care: a systematic review of tools for family medicine. Ann Fam Med 2011;9:155-64.

52 Brehaut JC, O'Connor AM, Wood TJ, et al. Validation of a decision regret scale. Med Decis Making 2003;23:281-92.

53 Pochard F, Azoulay E, Chevret S, et al. Symptoms of anxiety and depression in family members of intensive care unit patients: ethical hypothesis regarding decision-making capacity. Crit Care Med 2001;29:1893-7.

54 Herrmann C. International experiences with the Hospital Anxiety and Depression Scale--a review of validation data and clinical results. $J$ Psychosom Res 1997;42:17-41.

55 Zigmond AS, Snaith RP. The hospital anxiety and depression scale. Acta Psychiatr Scand 1983;67:361-70.

56 Excellence NIfHaC. Rehabilitation after critical illness, 2009. Available: https://www.nice.org.uk/guidance/cg83 [Accessed 6 July 2017].

57 Cameron IM, Crawford JR, Lawton K, et al. Psychometric comparison of PHQ-9 and HADS for measuring depression severity in primary care. $\mathrm{Br} J$ Gen Pract 2008;58:32-6.

58 Hinz A, Zweynert U, Kittel J, et al. [Measurement of change with the Hospital Anxiety and Depression Scale (HADS): sensitivity and reliability of change]. Psychother Psychosom Med Psychol 2009;59:394-400.

59 Sundin EC, Horowitz MJ. Impact of event scale: psychometric properties. Br J Psychiatry 2002;180:205-9.

60 Katz S, Ford AB, Moskowitz RW, et al. Studies of illness in the aged. The index of ADL: a standardized measure of biological and psychosocial function. JAMA 1963;185:914-9.

61 Kahn JM, Rubenfeld GD, Rohrbach J, et al. Cost savings attributable to reductions in intensive care unit length of stay for mechanically ventilated patients. Med Care 2008;46:1226-33.

62 Unroe M, Kahn JM, Carson SS, et al. One-Year trajectories of care and resource utilization for recipients of prolonged mechanical ventilation: a cohort study. Ann Intern Med 2010;153:167-75

63 Hussey MA, Hughes JP. Design and analysis of stepped wedge cluster randomized trials. Contemp Clin Trials 2007;28:182-91.

64 Thompson JA, Fielding KL, Davey C, et al. Bias and inference from misspecified mixed-effect models in stepped wedge trial analysis. Stat Med 2017;36:3670-82.

65 Hedeker D, Gibbons RD. Application of random-effects patternmixture models for missing data in longitudinal studies. Psychol Methods 1997;2:64-78.

66 White DB, Angus DC, Shields AM, et al. A randomized trial of a family-support intervention in intensive care units. N Engl J Med 2018;378:2365-75

67 Curtis JR, Downey L, Back AL, et al. Effect of a patient and clinician Communication-Priming intervention on patient-reported Goals-ofCare discussions between patients with serious illness and clinicians: a randomized clinical trial. JAMA Intern Med 2018;178:930.

68 Back AL, Arnold RM, Baile WF, et al. Approaching difficult communication tasks in oncology. CA Cancer J Clin 2005;55:164-77.

69 Brehaut JC, Graham ID, Wood TJ, et al. Measuring acceptability of clinical decision rules: validation of the Ottawa acceptability of decision rules instrument (OADRI) in four countries. Med Decis Making 2010;30:398-408.

70 Embriaco N, Azoulay E, Barrau K, et al. High level of burnout in intensivists: prevalence and associated factors. Am J Respir Crit Care Med 2007;175:686-92

71 Poncet MC, Toullic P, Papazian L, et al. Burnout syndrome in critical care nursing staff. Am J Respir Crit Care Med 2007;175:698-704.

72 Maslach C, Schaufeli WB, Leiter MP. Job burnout. Annu Rev Psychol 2001:52:397-422. 